\title{
Right-sided invasive metastatic thymoma of the heart
}

\author{
P. M. van der Zee • J. van Schuppen • \\ R. B. A. van de Brink
}

Published online: 13 April 2011

(C) The Author(s) 2011. This article is published with open access at Springerlink.com

\begin{abstract}
Cardiac tumours may display diverse symptoms through potential involvement of any structure of the heart. We describe a case of a highly malignant thymoma with involvement of different cardiac structures with important haemodynamic compromise. With the high sensitivity of transthoracic echocardiography for detection of intracardiac masses, computed tomography and magnetic resonance add essential structural preoperative information on the tumour and surrounding tissue as vessels, pleura, lung and mediastinum.
\end{abstract}

Keywords Cardiac tumor · Echocardiography ·

Cardiac CT . Thymoma Cardiac imaging

\section{Introduction}

In heart tumours, a variety of symptoms can be found, depending on the cardiac structures involved. Pericardial involvement occurs in approximately two-third of cases, which can lead to effusion and tamponade. Epicardial and myocardial localisation are each found in a third of cardiac

P. M. van der Zee $(\bowtie) \cdot R$. B. A. van de Brink Department of Cardiology, Academic Medical Center, room B2-223, Meibergdreef 9, 1105 AZ Amsterdam, the Netherlands

e-mail: p.m.vanderzee@amc.uva.nl

J. van Schuppen

Department of Radiology, Academic Medical Center, Meibergdreef 9 ,

1105 AZ Amsterdam, the Netherlands metastases, whereas endocardial localisation is rare $(5 \%$ of cases) [1].

As found in obduction series, metastatic tumours in the heart are much more common than primary cardiac tumours [1]. Haematogenous and lymphatogenous spread from intrathoracic tumours (lung cancer, breast carcinoma, and mesothelioma) or haematological malignancies (leukaemia and lymphoma) are most frequent, while direct extension has been described primarily from thymoma and oesophageal cancer [1]. Whereas most thymomas with cardiac involvement are limited to the pericardium [2], cases of extension in the caval vein and right atrium have also been described [3, 4]. We describe a case of a highly malignant thymoma with involvement of different cardiac structures with important haemodynamic compromise.

\section{Case report}

A 25-year-old Nigerian man presented to our emergency department with complaints of tiredness, night sweats, and dizziness on exertion. On clinical examination the patient had facial swelling and venous distension in the neck. Blood pressure was $135 / 77 \mathrm{mmHg}$ with pulsus paradoxus. Cardiac auscultation was normal. The liver was enlarged. There was no peripheral oedema. Electrocardiography showed sinus tachycardia of 110 beats/min, a vertical axis, QS pattern in V1 and a Q wave in V2 and low voltages in the limb leads. Laboratory testing showed lymphocytosis, increased creatinine $(120 \mathrm{mmol} / \mathrm{l})$ and abnormal liver enzymes (total bilirubin $47 \mu \mathrm{mol} / \mathrm{l}$, aspartate aminotransferase $124 \mathrm{U} / \mathrm{l}$, alanine aminotransferase $200 \mathrm{U} / 1$ and $\gamma$ - 
glutamyl transferase $268 \mathrm{U} / 1)$. On chest X-ray, right pleural effusion and mediastinal widening were seen. With abdominal ultrasound the liver diameter was $17.5 \mathrm{~cm}$, with ascites, pleural and pericardial effusion and hepatopetal flow in the portal vein. Transthoracic echocardiography revealed a mass almost completely occupying the right atrium and ventricle with a distended inferior vena cava (Fig. 1). The pressure gradient over the tricuspid valve was $25 \mathrm{mmHg}$ (PAP 25+15 mmHg). Pericardial effusion was present. Computed tomography (CT) of the thorax with contrast injected into the right antecubital vein revealed a mass in the anterior mediastinum with no clear distinction from the surrounding structures. There was a lack of contrast in the innominate and superior caval veins, and contrast filled the right atrium through the inferior caval vein by way of collateral veins. A large right atrial filling defect was found with faint filling of the right ventricle (Fig. 2).

Due to the important haemodynamic compromise, the induction of anaesthesia was possible only after initiation of cardiopulmonary bypass with cannulation of femoral vessels under local anaesthesia. A large mass was removed from the right atrium $(7 \times 5 \times 5 \mathrm{~cm})$, but tumour tissue extending into the superior vena cava was only partially removed. Histology was compatible with type B2 thymoma. Moreover, within the tumour, immature T cells were found, with positive CD4, CD8, CD5, TdT and cytoplasmatic CD3 immunostaining. After an uneventful recovery, the patient was referred for palliative chemotherapy with cisplatinum, adriamycin and cyclophosphamide. Six months later, after four cycles of chemotherapy, regression of the residual tumour was seen and additive radiotherapy was planned.

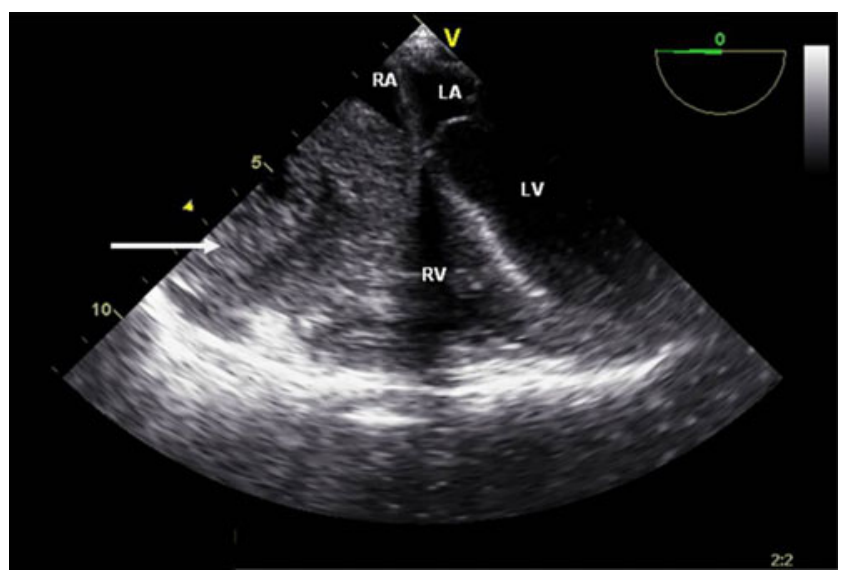

Fig. 1 Four-chamber view of the intracardiac mass on transthoracic echocardiography. The arrow indicates the mass in the right ventricle. $\mathrm{RA}$, right atrium. RV, right ventricle. LA, left atrium. LV left ventricle

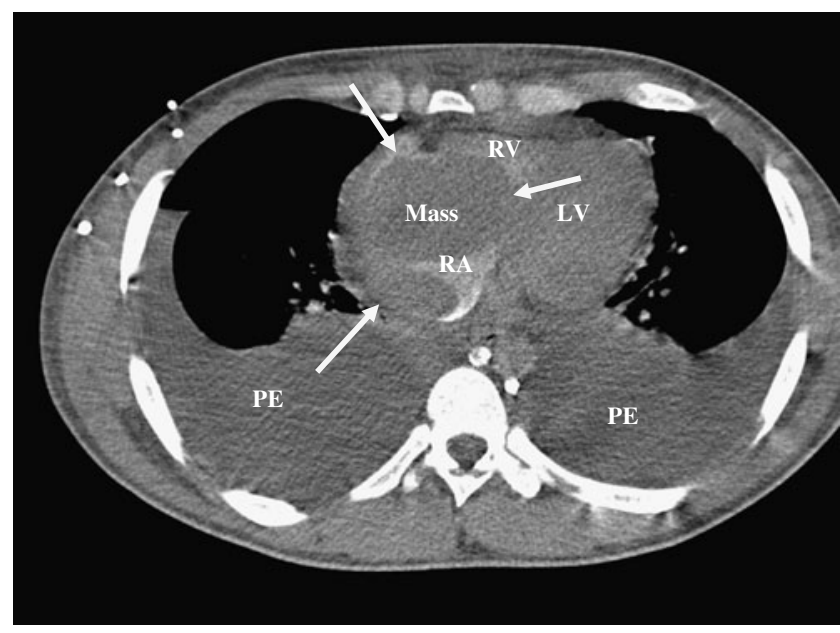

Fig. 2 Axial computed tomography at the level of the heart. The arrows indicate the mass in the right atrium and ventricle. The mass is surrounded with contrast. Note the faint contrast distribution around the tumour and the filling of collateral veins such as the superficial thoracic veins, the azygos and hemiazygos veins and the ipsilateral mammary vein. Pleural effusion on both sides. RV, right ventricle; LV, left ventricle; RA, right atrium; PE, pleural effusion

\section{Discussion}

Independent of the nature of the primary tumour, cardiac metastases can involve any structure of the heart [1]. The clinical presentation may therefore vary considerably, and imaging will be prompted to provide the correct diagnosis. Sensitivity of transthoracic echocardiography for the detection of intracardiac masses has been reported $93 \%$ [5].

$\mathrm{CT}$ and magnetic resonance (MR) offer a structural overview of the tumour and surrounding tissue as vessels, pleura, lung and mediastinum. Extension in surrounding tissue and possible metastasis can be evaluated for preoperative information. Intravascular contrast provides information about intracardial extension and flow around the tumour. [3]

Furthermore, cardiac MR can provide a differentiation between tumour, myocardium, thrombus and blood flow artifacts. MR can characterise tumour by different signal intensities on $\mathrm{T} 1$ and $\mathrm{T} 2$, and different enhancement after contrast, where most tumours have low intensity on $\mathrm{T} 1$, high intensity on $\mathrm{T} 2$ and strong enhancement after contrast. Our patient presented with cardiovascular collapse due to obstruction of the superior caval vein, right atrium and tricuspid valve. The imaging modalities of transthoracic echocardiography and CT provided a prompt evaluation of the severity and extent of the obstruction and offered essential pre-operative information on tumour extension and potential metastases. 
Open Access This article is distributed under the terms of the Creative Commons Attribution Noncommercial License which permits any noncommercial use, distribution, and reproduction in any medium, provided the original author(s) and source are credited.

\section{References}

1. Bussani R, De-Giorgio F, Abbate A, et al. Cardiac metastases. J Clin Pathol. 2007;60:27-34.
2. Kondo K, Monden Y. Lymphogenous and hematogenous metastasis of thymic epithelial tumors. Ann Thorac Surg. 2003;76:1859-64.

3. Restrepo CS, Largoza A, Lemos DF, et al. CT and MR imaging findings of malignant cardiac tumors. Curr Probl Diagn Radiol. 2005;34:1-11.

4. Dursun M, Sarvar S, Cekrezi B, et al. Cardiac metastasis from invasive thymoma. Cardiovasc Intervent Radiol. 2008;31 Suppl 2:S209-12.

5. Meng Q, Lai H, Lima J, et al. Echocardiographic and pathologic characteristics of primary cardiac tumors: a study of 149 cases. Int J Cardiol. 2002;84:69-75. 